ARTICLE

\title{
The PTSim and TOPAS Projects, Bringing Geant4 to the Particle Therapy Clinic
}

\author{
Takashi AKAGI ${ }^{1}$, Tsukasa ASO ${ }^{2}$, Bruce FADDEGON ${ }^{3}$, Akinori KIMURA $^{4}$, Naruhiro MATSUFUJI ${ }^{5}$, Teiji NISHIO ${ }^{6}$, \\ Chihiro OMACHI ${ }^{7}$, Harald PAGANETTI ${ }^{8}$, Joseph PERL ${ }^{9}$, Takashi SASAKI ${ }^{7, *}$, Daren SAWKEY ${ }^{3}$, Jan SCHÜMANN ${ }^{8}$, \\ Jungwook SHIN ${ }^{3}$, Toshiyuki TOSHITO ${ }^{10}$, Tomohiro YAMASHITA ${ }^{1}$ and Hajime YOSHIDA ${ }^{11}$ \\ ${ }^{1}$ Hyogo Ion Beam Medical Center, 1-2-1, Kouto, Shingu-cho, Ibo-gun, Hyogo, 679-5165, Japan \\ ${ }^{2}$ Toyama National College of Technology, 1-2 Ebie-Neriya, Imizu, Toyama, 933-0293, Japan \\ ${ }^{3}$ University of California San Francisco, San Francisco, CA, 94143-1708, USA \\ ${ }^{4}$ Ashikaga Institute of Technology, 268-1, Omae-cho, Ashikaga, Tochigi, 326-8558, Japan \\ ${ }^{5}$ National Institute for Radiology Science, 4-9-1, Anagawa, Inage-ku, Chiba-shi, Chiba, 263-8555, Japan \\ ${ }^{6}$ Cancer Center Hospital East, 6-5-1, Kashiwanoha, Kashiwa, Chiba, 277-8577, Japan \\ ${ }^{7}$ KEK, 1-1 Oho, Tsukuba, Ibaraki, 305-0801, Japan \\ ${ }^{8}$ Massachusetts General Hospital, Boston, MA, 02114, USA \\ ${ }^{9}$ SLAC, 2575 Sand Hill Road, Menlo Park, CA, 94303, USA \\ ${ }^{10}$ Nagoya City, 1-1, Sannomaru 3-chome, Naka-ku, Nagoya, 460-8508, Japan \\ ${ }^{11}$ Shikoku University, Ojin-cho, Tokushima-shi, Tokushima, 771-1192, Japan
}

\begin{abstract}
Though the Geant4 Simulation Toolkit has been widely accepted in the particle therapy community, with research and clinical use at most of the major centers currently involved in this innovative approach to cancer treatment, the high level of Geant4 expertise required for these applications has proven a serious barrier for users. The PTSim collaboration in Japan and the TOPAS collaboration in the United States wrap and extend the Geant4 toolkit to meet the needs of this critical community. PTSim has provided a common platform to model three Japanese proton and ion therapy facilities plus three more in other countries, allowing users who are not Geant4 experts to accurately and efficiently run Geant 4 simulations for any of these pre-built configurations. Building on a rich history of proton therapy applications at MGH (site of the world's first proton therapy system), NCC Korea, and elsewhere, the TOPAS project aims to take flexibility further, allowing any particle therapy clinician or researcher to Geant4-simulate their own real or envisioned facility still without requiring a Geant 4 expert. We describe these projects, how their designs bridge the gap between flexibility and ease of use, what key missing software components they have contributed and how the two projects may evolve together.
\end{abstract}

KEYWORDS: particle therapy, ion therapy, carbon therapy, proton therapy, Monte Carlo, simulation, Geant4

\section{Introduction}

Geant $4^{1,2)}$ is a software toolkit to simulate the interaction of particles in matter. It has been widely used in various fields from high energy physics (HEP) to nuclear physics to space and medicine. A key area for Geant4 in medical physics has been particle therapy, radiation therapy performed with protons or heavy ions.

Particle therapy promises improved treatment and reduced side effects for many cancers compared with other therapeutic options such as $\mathrm{x}$-ray or surgery. Thirty-five particle therapy centers are currently in operation world wide, with at least twenty-five more currently in planning or construction. ${ }^{3)}$ Monte Carlo simulation can be helpful in design of such treatment facilities and in the comparison of treatment plans. Such Geant4 simulations have been carried out at many institutions with good agreement to measurement. ${ }^{4,5)}$

While Geant 4 has been heavily used in medical physics research, applications on the clinical side are limited by issues of computation speed and, more importantly, the level

*Corresponding author, E-mail:Takashi.Sasaki@kek.jp

(C) 2011 Atomic Energy Society of Japan, All Rights Reserved. of Geant4 expertise required to perform these simulations. Key differences between the needs of clinical medical physics and those of Geant4's original user base, high energy physics, result in needs not met by the already rich toolkit of Geant4.

In parallel with improvements to accuracy and speed within Geant4 itself, groups of Geant4 developers and medical physicists have formed focused projects to address the specific needs of this community for reliability, repeatability, geometry, accuracy, speed, functionality and ease of use.

This paper describes two such efforts, one in Japan, PTSim (Particle Therapy Simulation), ${ }^{6}$ ) and another in the United States, TOPAS (TOol for PArticle Simulation). Both projects are designed along principles of Object-Oriented technology, are implemented like Geant4 in the language $\mathrm{C}++$ and have committed to make their software freely available. The goal is that all users of particle therapy facilities, researchers and clinicians, should be able to exploit Monte Carlo simulation with improved reliability, repeatability and ease of use. 
Table 1 Institutions Sampled for PTSim Use Cases

\begin{tabular}{lll}
\hline Institution & Location & Particle \\
\hline $\begin{array}{l}\text { National Institute of Radi- } \\
\text { ological Science (NIRS) }\end{array}$ & Chiba, Japan & carbon \\
\hline $\begin{array}{l}\text { National Cancer Center } \\
\text { East Hospital (NCC-East) }\end{array}$ & $\begin{array}{l}\text { Kashiwa, } \\
\text { Japan }\end{array}$ & proton \\
\hline $\begin{array}{l}\text { Hyogo Ion Beam Medical } \\
\text { Center(HIBMC) }\end{array}$ & $\begin{array}{l}\text { Hyogo, Ja- } \\
\text { pan }\end{array}$ & $\begin{array}{l}\text { proton } \\
\text { and car- } \\
\text { bon }\end{array}$ \\
\hline $\begin{array}{l}\text { University of California } \\
\text { San Francisco (UCSF) }\end{array}$ & $\begin{array}{l}\text { San Fran- } \\
\text { cisco, USA }\end{array}$ & $\begin{array}{l}\text { proton } \\
\text { (UC } \\
\text { Davis) }\end{array}$ \\
\hline $\begin{array}{lll}\text { German Cancer Research } \\
\text { Center(DKFZ) }\end{array}$ & $\begin{array}{l}\text { Heidelberg, } \\
\text { Germany }\end{array}$ & $\begin{array}{l}\text { carbon } \\
\text { (GSI) }\end{array}$ \\
\hline CATANA at INFN Catania & $\begin{array}{l}\text { Catania, Ita- } \\
\text { ly proton }\end{array}$ & \\
\hline $\begin{array}{l}\text { Proton Medical Research } \\
\text { Center at U. of Tsukuba }\end{array}$ & $\begin{array}{l}\text { Tsukuba, } \\
\text { Japan }\end{array}$ & proton \\
\hline
\end{tabular}

\section{PTSim Overview}

PTSim has been developed in the project, "Development of simulation framework for advanced radio therapy", funded by the Japan Science and Technology Agency (JST) in the program of Core Research for Evolutional Research and Technology(CREST), October 2003 to March 2010. This joint project among Geant4 developers, physicists and medical physicists produced a software suite for simulating particle therapy with a special focus on carbon therapy. Efforts on further development of PTSim are still under way to include more functionality and improve the performance.

The design of PTSim was based on use-cases sampled from medical physicists at the treatment facilities shown in Table 1. PTSim includes a class library for geometry description, material definition, optimized physics process setting (PhysicsList in the Geant4 context), scorers, event level parallel processing and the main program.

\section{TOPAS Overview}

Building on a rich history of Geant4 proton therapy applications at Massachusetts General Hospital (MGH, site of the worlds first proton therapy system), the National Cancer Center Korea (NCC), ${ }^{7)}$ and elsewhere, the US National Institutes of Health-funded TOPAS project aims to take flexibility further. With TOPAS, any particle therapy clinician or researcher will be able to Geant4-simulate their own real or envisioned facility (modify an existing design, create a whole new one) still without requiring a Geant4 expert. TOPAS adds advanced I/O facilities for "phase space files", extends handling for patient scan data, phantoms and dosimetry devices, and provides a comprehensive "sequence management" system to handle the many time-dependent aspects of a particle-therapy setup (parts that physically move during treatment, such as modulator wheels or range shifters, fields that vary during treatment, such as for

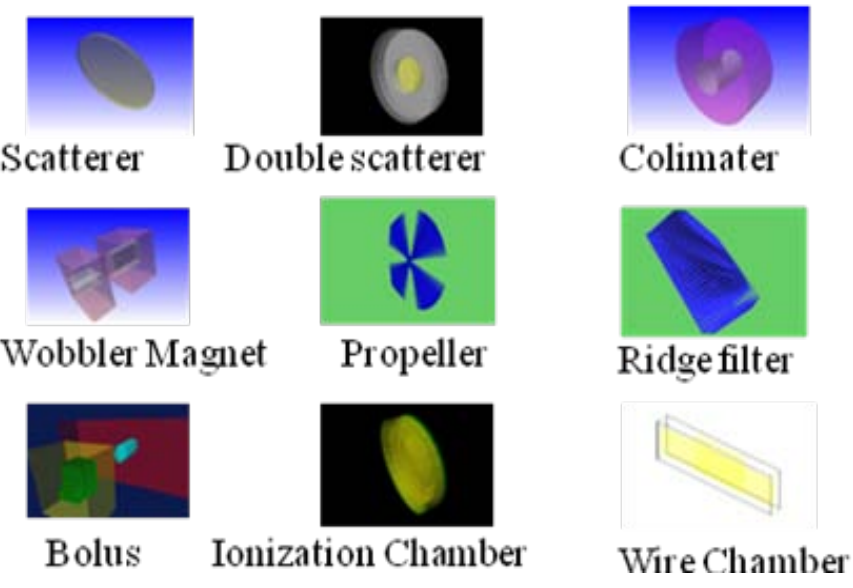

Fig. 1 Example PTSim beam line components

scanned beam treatments, or other time-varying quantities, such as beam current that is modulated during treatment). Like PTSim, TOPAS assures relevance to its user domain by teaming Geant4 developers and medical physicists in a single close collaboration.

\section{Geometry}

Both PTSim and TOPAS take the point of view that the medical physics user should not need to be an expert in Geant4 geometry description. While the geometry capabilities of Geant 4 are significant, they can be confusing for new users. PTSim and TOPAS provide easier ways to describe geometry (while still allowing the user to use the full Geant4 $\mathrm{C}++$ ). The PTSim user can access a library of already created beamline components and can adjust them from macro commands. The TOPAS user has similar capabilities configurable from control files.

PTSim and TOPAS are developed with the understanding that most users will not need to re-implement the geometry descriptions of a complete treatment facility. Rather, they will take an implementation from a previous user and then apply slight changes of their own. We therefore provide easy ways to share implementations between users.

In medical physics applications, we cannot rely on constructive solid geometry for all of the geometry as done in HEP. Geometry from DICOM files (Digital Imaging and Communications in Medicine, from the National Electrical Manufacturers Association ${ }^{8)}$ ) and CAD (Computer Aided Design) play significant roles described below.

\section{Beam Line Geometry}

Through a study of many potential users, PTSim identified that many of sampled facilities have common components in their beam lines. PTSim provides abstract classes for these common components such that users can implement their own specific components by inheriting from these abstract classes. The most difficult to implement but most important components in a beam line are lateral beam spreading systems and range modulators. The base classes 


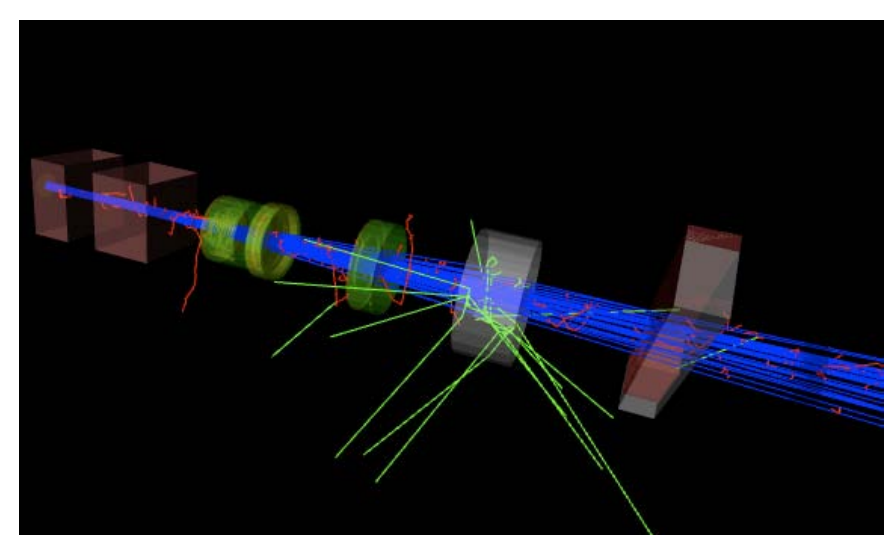

Fig. 2 Example beam line with simulated particles

for Wobbler magnets, scatterers, Ridge filters, propeller blades, beam scanning magnet and so on are provided within PTSim to make the implementation of such components easier (see Fig. 1).

After the individual components have been implemented, their positions and angles relative to the beam line are adjusted. Figure 2 shows a PTSim example of a beam line with simulated particles.

The TOPAS project encapsulates component designs from earlier Geant4 implementations of the MGH and KNCC proton therapy beamlines. Future improvements to TOPAS will include the ability to import components from CAD. Such capabilities, originally developed for the Geant4 space user community are now being used for import of beam line components. ${ }^{9)}$

\section{Patient Geometry}

Hospital information systems typically provide patient scan information, from CT or MRI, in DICOM format. The DICOM represents the patient image as a contiguous set of voxels formed into a box (of typical dimensions, $512 \times 512$ $\times 256$ voxels, with each voxel on the order of a half $\mathrm{mm}$ on a side). A scalar for each voxel represents the scanned value in that voxel. Because the overall DICOM forms a box, it is common for the DICOM to include both the patient and some amount of air around the patient. PTSim and TOPAS both include code to import such information and to automatically convert DICOM density information (Hounsfield units) into material information (composition and density).$^{10,11)}$

PTSim also includes the capability to process the additional information present in the Radio Therapy extensions (RT) of the DICOM-RT format. This provides additional information about treatment settings (beam and patient angles, etc.) for each patient but is typically specialized to only a specific institution (the format contains a large amount of user-defined information). Thus while DICOM is well established, usage of DICOM-RT is still limited. Among facilities sampled by PTSim, RT is used only by HIBMC and the PTSim DICOM-RT interface is therefore specialized for HIBMC.

\section{Geometry Uncertainty}

Accuracy of geometry models in medical applications can be limited by manufacturer tolerance and the proprietary confidential nature of some aspects of medical equipment design. Where fully accurate designs are unavailable, the user is forced to rely on simplified drawings and with limited observation of the relevant components. Such uncertainties must be handled by the addition of free parameters to the models. ${ }^{12)}$

\section{Geometry Overlaps}

Another unique aspect of geometry for medical physics applications is the potential for overlap between parts of the treatment apparatus and parts of the patient geometry. Recall that the patient is represented in the DICOM format as a box, including some of the air around the patient. Because most particle therapy treatments bring the final parts of the beam delivery system very close to the patient, some parts of that beam delivery system may overlap some of the air parts of the DICOM. This can be dealt with by trimming back these air regions, but a more common solution is to mediate the calculation through a phase space file. In this technique, the output of the beam delivery simulation is recorded at a surface (generally a plane just after the beam delivery system), and this list of stored particles is then replayed from that plane through the patient.

\section{Motion}

Particle therapy involves many moving and otherwise time-dependent parts. A key component of many particle therapy systems, the range modulator wheel, moves throughout the treatment to change the depth penetration of the particles in the patient. Beam current may also be modulated during the treatment. Other treatment head components may move in or out of field during treatment to change scatter or collimation. The entire treatment gantry and/or patient couch may move to adjust the beam angle. Scanned beam therapy includes time-dependent magnetic fields to steer the beam. ${ }^{13,14)}$ Finally, the patient is always moving, simply through breathing and gradual shifting of organs. ${ }^{15)}$ PTSim accommodates much of this and TOPAS goes further to a comprehensive "sequence management" system.

\section{Output Geometry}

Visualization of patient geometry presents its own challenges to Geant4. While Geant4 already contained a wide variety of visualization drivers for investigation and presentation of the most complex constructive solid geometry, ${ }^{16)}$ there were no tools to adequately represent the volumetric information of DICOM input or patient dose output.

To address this issue, PTSim developed a novel DICOM and volume data visualization system called gMocren. ${ }^{17,18)}$ The gMocren system can represent DICOM outputs from multi vendor CTs and overlay additional Geant4 data such as treatment head geometry, particle trajectories and calculated dose in an integrated, highly interactive visualization. 


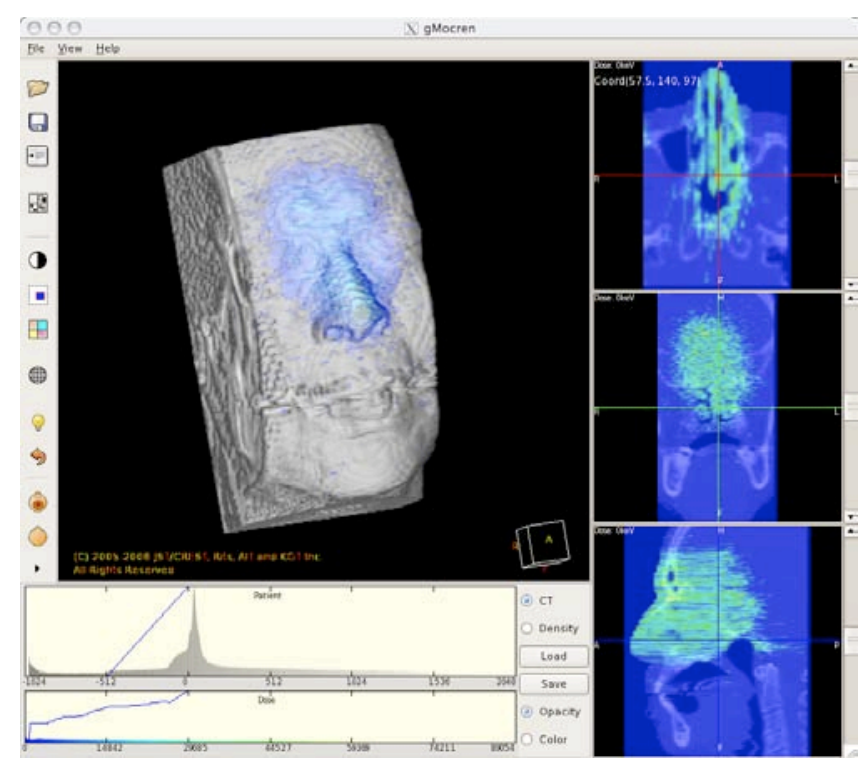

Fig. 3 gMocren image of dose overlaid on DICOM image

Figure 3 shows a Geant4 dose distribution simulation overlaid on a DICOM image. The gMocren client has been made freely available to all Geant4 users, with a corresponding volume data interface built into the Geant4 visualization system.

\section{Accuracy and Validation}

Accuracy requirements for particle therapy are on the order of a few percent. If Monte Carlo is much less accurate than that, users will instead opt for faster parameterized methods. Validation of simulation results against measurements has been done for protons and carbon ions. After improving Geant4 electromagnetic and hadron physics (in concert with the larger Geant4 community), PTSim reproduces dose profiles of therapeutic carbon and proton beams measured in water phantoms within the required accuracy in radiotherapy. ${ }^{19-21)}$ TOPAS collaborators from MGH have achieved similar results. ${ }^{22)}$

\section{Calculation Time Requirements}

Monte Carlo is inherently calculation-time intensive. A typical head and neck dose calculation at MGH requires 240 hours of running on a standard $2 \mathrm{GHz}$ PC (run overnight as 20 jobs of 12 hours each). While simulation production in HEP may involve thousands of processors running continuously for many months, usage patterns for clinical medical physics follow more of a burst pattern. Running for $15 \mathrm{mi}-$ nutes on a thousand processors is more useful than a thousand minutes on 15 processors. PTSim includes a parallel execution option using MPI (Message Passing Interface) $^{23)}$ and also SAGA (Simple API for Grid Applications). ${ }^{24)}$ TOPAS will include similar kinds of tools to simplify management of large numbers of parallel jobs suitable for cluster, grid and cloud solutions.

\section{Usability}

Because it is an extremely general purpose toolkit, and because its original user community, HEP, involves large collaborations that can spend significant personnel resources on simulation development, Geant4 emphasizes flexibility. It is the role of applications such as PTSim and TOPAS to wrap Geant4 into something easier to use for a particular user community. In clinical medical physics, usability means reliability, and repeatability as much as ease of use.

\section{Reliability}

PTSim and TOPAS give the user pre-built sets of particle therapy beamline components, already validated against experimental data. They incorporate proven solutions for patient data import and validated sets of physics options. TOPAS will go on to add principles from Integrated Safety Management, in particular the use of Engineering versus Administrative Controls. The former means that the user will be specifically locked out from certain unreliable practices. For example, if any key setting is changed by the user in the middle of a run (something one may want to do for test purposes only), the code will automatically tag this run as "test only" and will refuse to send output to the standard output area.

\section{Repeatability}

TOPAS will incorporate the concept of Data Provenance, which tags a given result with all of the information that explains how that result was obtained. This accommodates the natural working method of the medical physicist, to change just one variable at a time, rerun the simulation and compare the results.

\section{Ease of Use}

Geant4's great flexibility is known to come with a steep learning curve. Domain-specific applications such as PTSim and TOPAS can help, providing examples of applications that are already close to the user's final requirements.

Among the features PTSim has contributed back to the Geant4 distribution to make Geant4 easier to use is a simplified scoring system. Geant4's original users were content to each develop their own sensitive detectors (detector development was a core Geant4 use case). Medical users on the other hand may simply wish to score standard quantities (dose, energy, etc.) in a simple surface or volume. The new scoring system now included in all Geant4 distributions allows the user to define scoring surfaces and volumes from $\mathrm{C}++$ code or even from simple commands.

Both PTSim and TOPAS provide example applications that are already close to the user's final requirements. PTSim provides the user a library of pre-built particle therapy beamline components. TOPAS also includes such components, and adds an easy way for the user to modify such components through control files. The TOPAS architecture allows users to modify existing components or model new ones and then easily share these models with collaborators. 
TOPAS has added an easy to use phase space input/output module so that the set of particles passing through a given surface can be easily written out to a standard, IAEA-compliant, file format and then later read back in as new primary particles.

In medical physics, there are times when one wishes to calculate dose as if the body were just water, and other times where one wishes to calculate dose as if the body were its full set of complex materials. TOPAS will calculate dose in both ways. This feature is particularly important for particle therapy, given the heavy dependence of proton and carbon dose on material. ${ }^{25,26)}$ TOPAS will extend its patient-import code to also handle various forms of computational phantoms $^{27)}$ and various common dosimetry devices.

\section{Conclusion}

The concepts employed in PTSIM and TOPAS have proven practical for proton beam simulations in radiotherapy. PTSim has been used at many treatment facilities including NIRS, NCC-East, HIBMC and etc. TOPAS has been used to model the IBA and STAR beamlines at MGH's Francis $\mathrm{H}$ Burr Proton Center, the proton therapy eye treatment line operated by UCSF at UC Davis, and will be opening to selected alpha testers in the fall of 2011. Because PTSim will soon be moving to the Apache software license ${ }^{28)}$ with liberal terms similar to those already in the TOPAS project plan, the two teams anticipate strong sharing of components in the near future.

\section{Acknowledgments}

The authors would like to thank members of the Geant 4 collaboration, especially, Makoto Asai, Dennis Wright and Tatsumi Koi of the SLAC National Accelerator Laboratory and Vladimir Ivanchenko of the EMSU Lomonosov Moscow State University for their great help in the development of our software. We also thank Prof. Oliver Jäckel of DKFZ and Prof. Takeji Sakae of Univ. of Tsukuba for the valuable discussion on the PTSim design. PTSim was supported by the Japan Science and Technology Agency in the program of Core Research for Evolutional Research and Technology. TOPAS is supported by the US National Institutes of Health under contract number 1R01CA140735-01.

\section{References}

1) S. Agostinelli et al., "Geant4, a simulation toolkit,” Nucl. Instr. Meth. Phys. Res., A506, 250-303 (2003).

2) J. Allison et al.,"Geant4 Developments and Applications," IEEE Trans. Nucl. Sci., 53, no. 1,270-278 (2006).

3) Proton Therapy Co-Operative Group, http://ptcog.web.psi.ch/

4) H. Paganetti, "Monte Carlo method to study the proton fluence for treatment planning,” Med. Phys., 25, 2370-2375 (1998).

5) H. Paganetti, H. Jiang, K. Parodi, R. Slopsema, M. Engelsman, "Clinical implementation of full Monte Carlo dose calculation in proton beam therapy,” Phys. Med. Biol., 53[17] 4825-4853 (2008).

6) T. Sasaki, S. Tanaka et al., "Development of simulation framework for advanced radio therapy,” Simulation, 28[1] (feature articles), 2-40 (2009), [in Japanese].

7) J. Shin, D. Kim, Y.K. Lim et al., "Monte Carlo Modeling and Simulation of a Passive Treatment Proton Beam Delivery system using a Modulation Wheel," J. Korean Phys. Soc., 56, p 153-163 (2010).

8) DICOM, http://medical.nema.org/

9) M. Constantin, D. Constantin, P. Keall, A. Narula, M. Svatos, J. Perl, "Linking Computer-Aided Design (CAD) to Geant4-based Monte Carlo Simulations for Precise Implementation of Complex Treatment Head Geometries", Phys. Med. Biol., 55, N211 (2010).

10) H. Jiang, H. Paganetti, "Adaptation of GEANT4 to Monte Carlo dose calculations based on CT data," Med. Phys., 31, 2811-2818 (2004).

11) H. Jiang, J. Seco, H. Paganetti, "Effects of Hounsfield number conversions on patient CT based Monte Carlo proton dose calculation,” Med. Phys., 34, 1439-1449 (2007).

12) B. Faddegon, J. Perl, M. Asai, "Monte Carlo Simulation of Large Electron Fields," Phys. Med. Biol., 53, 1497-1510 (2008).

13) S. W. Peterson, J. Polf, M. Bues, G. Ciangaru, L. Archambault, S. Beddar, A. Smith, "Experimental validation of a Monte Carlo proton therapy nozzle model incorporating magnetically steered protons,” Phys. Med. Biol., 54, 3217-3229 (2009).

14) S. Peterson, J. Polf, G. Ciangaru, S.J. Frank, M. Bues, M, Smith A. "Variations in proton scanned beam dose delivery due to uncertainties in magnetic beam steering," Med. Phys., 36[8), 3693-3702 (2009).

15) H. Paganetti, H. Jiang, A. Trofimov, “4D Monte Carlo simulation of proton beam scanning: Modelling of variation in time and space to study the interplay between scanning pattern and time-dependent patient geometry," Phys. Med. Biol., 50 983-990 (2005).

16) J. Allison, M. Asai, G. Barrand, M. Donszelmann, K. Minamimoto, J. Perl, S. Tanaka, E. Tcherniaev, J. Tinslay, "The Geant4 Visualisation System,” Comput. Phys. Comm., 178[5], 331-365 (2008).

17) A. Kimura, S. Tanaka, T. Sasaki., "A visualization tool for Geant4-based medical physics applications,” Int. J. Comput. Assist. Radiol. Surg., 1 462-463 (2006).

18) A. Kimura, A. Saitoh, T. Sasaki, S. Tanaka,"gMocren: a visualization tool for Geant medical applications,” Int. J. Comput. Assist. Radiol. Surg., 2, 459-460 (2007).

19) T. Aso, A. Kimura, S. Tanaka, H. Yoshida, N. Kanematsu, T. Sasaki, T. Akagi, "Verification of the Dose Distributions with Geant4 Simulation for Proton Therapy," IEEE Trans. Nucl. Sci., 52[4], 896-901 (2005).

20) S. Kameoka et al., "Dosimetric evaluation of nuclear interaction models in the Geant4 Monte Carlo simulation toolkit for carbon-ion radiotherapy,” Radiol. Phys. Technol., 1, 183-187 (2008).

21) Y. Kase et al., "Biological dose calculation with Monte-Carlo physics simulation for heavy-ion radiotherapy," Phys. Med. Biol., 51[24] N467-N475 (2006).

22) C. Zacharatou Jarlskog, H.Paganetti, "Physics settings for using the Geant4 toolkit in proton therapy," IEEE Trans. Nucl. Sci., 55, 1018-1025 (2008).

23) MPI, http://www.mpi-forum.org/

24) SAGA, http://saga.cct.lsu.edu/

25) H. H. Liu, P. Keall, "Dm rather than Dw should be used in Monte Carlo treatment planning,” Med. Phys., 29, 922-924 
(2002).

26) H. Paganetti, "Dose to water versus dose to medium in proton beam therapy,” Phys. Med. Biol., 54, 4399-4421 (2009).

27) H. Paganetti, "The use of computational patient models to as- sess the risk of developing radiation induced cancers from radiation therapy of the primary cancer," Proc. of the IEEE, 97, 1977-1987 (2009).

28) Apache software license, http://www.apache.org/licenses/ 